\title{
Acalasia y su relación con el cáncer esofágico
}

\author{
Attila Csendes J. ${ }^{1}$, Javier Ignacio Toro P. ${ }^{1}$, Enrique Lanzarini S. ${ }^{1}$ y Manuel Figueroa G. ${ }^{1}$
}

Departamento de Cirugí Hospital Clínico Universidad de Chile. Santiago, Chile.

Recibido 2020-09-22 aceptado 2020-11-05

Correspondencia a: Dr. Attila Csendes J. acsendes@hcuch.cl

\section{Esophageal acalasia and its relationship with esophageal cancer}

Achalasia is a primary motor disorder of the esophageal smooth muscle characterized by dysphagia, pseudoregurgitation, and weight loss. Treatment can be endoscopic or surgical. The long-term results are only known from surgery, while endoscopic results still have a very short follow-up and do not allow us to draw valid conclusions. Achalasia is a lesion that has a significantly higher probability of developing esophageal cancer, whether of the epidermoid type, due to chronic inflammation and food retention in the esophagus, or an adenocarcinoma, secondary to gastroesophageal reflux, which appears after any treatment. Publications show that about 3 to $4 \%$ of patients present in time, about 10 to 15 years after treatment, the development of advanced cancer of the esophagus. It is concluded that clinical and endoscopic follow-up is essential in these patients on a routine basis.

Key words: acalasia; esophageal cancer; esophagomyotomy.

\section{Resumen}

La acalasia es un trastorno motor primario de la musculatura lisa esofágica que se caracteriza por disfagia, pseudorregurgitación y baja de peso. El tratamiento puede ser endoscópico o quirúrgico. Sólo se conocen los resultados a largo plazo de la cirugía, mientras que los endoscópicos tienen aún un seguimiento muy corto y no permiten sacar conclusiones valederas. La acalasia es una lesión que tiene una probabilidad significativamente mayor de desarrollar un cáncer esofágico, ya sea de tipo epidermoide, por inflamación crónica y retención de comida en el esófago, o un adenocarcinoma, secundario a reflujo gastroesofágico, que aparece posterior a cualquier tratamiento. Las publicaciones muestran que alrededor de $3 \%$ a $4 \%$ de los pacientes presentan a largo plazo, sobre 10 a 15 años postratamiento, el desarrollo de un cáncer avanzado del esófago. Se concluye que es indispensable un seguimiento clínico y endoscópico en forma rutinaria a estos pacientes.

Palabras clave: acalasia; cáncer de esófago; esofagomiotomía.

\section{Introducción}

La acalasia, que significa "falla en la relajación", es un trastorno crónico de la musculatura lisa esofágica, que se traduce en una falla de la relajación del esfínter esofágico inferior (EEI) hipertensivo y en la pérdida de una normal peristalsis, provocando la aparición de síntomas como disfagia, baja de peso y pseudorregurgitaciones ${ }^{1}$. Este esfínter está bajo control neuronal mediante el plexo de Auerbach que posee neuronas excitatorias como inhibitorias. En acalasia hay una destrucción total o parcial de las neuronas ganglionares, lo que ocasiona que el esfínter se relaje parcialmente con la deglución ${ }^{2}$. Se puede presentar a cualquier edad y en ambos sexos de manera igual, entre los 25 y 60 años (promedio
45 años), con una prevalencia de 1 en 100.000 personas $^{3}$. Se ha sugerido que la acalasia tiene un riesgo significativamente mayor (cerca de 40 veces) de desarrollar un cáncer esofágico comparado con la población normal ${ }^{4}$.

El objetivo de la presente revisión fue revisar la bibliografía nacional e internacional referente a la evidencia existente sobre la relación entre acalasia y cáncer esofágico.

\section{Materiales y Método}

Se revisaron las publicaciones referentes a "cáncer de esófago" "acalasia", "incidencia de cáncer esofágico en pacientes con acalasia", "carcinoma 
escamoso en acalasia esofágica", "adenocarcinoma esofágico en acalasia”, con filtro desde 1960 hasta la actualidad. Todas en lenguaje inglés y español publicadas en Pubmed y SCielo desde sus respectivas páginas web oficiales, con libre acceso a sus datos. Los manuscritos tenían que incluir una casuística de pacientes con acalasia sometidos a intervención quirúrgica y que hayan sido controlados a más de 5 años. Se incluyó referencias tanto laparotómicas y laparoscópicas.

\section{Resultados}

El objetivo del tratamiento de la acalasia es revertir o corregir la obstrucción funcional del EEI. Ningún tratamiento resuelve la pérdida neuronal, de modo que debe considerarse paliativa ${ }^{5,6}$. La cirugía para tratar la acalasia ha dado buenos resultados en numerosos estudios. Este abordaje comenzó con Heller en $1913^{7}$ mediante una esofagomiotomía anterior y posterior, disminuyendo los síntomas de cardioespasmo. Sin embargo, trajo como consecuencia un reflujo gastroesofágico permanente. Posteriormente, Zaaijer perfecciona la técnica en $1923^{8}$ con solamente una esofagomiotomía anterior, produciendo menores daños colaterales. Actualmente, la miotomía laparoscópica disminuye el tiempo de recuperación del paciente y atenúa los síntomas posoperatorios.

En 1872 se reportó el primer caso que relaciona la acalasia con el cáncer de esófago ${ }^{4}$. El cáncer esofágico puede ser producto de múltiples patologías, tales como hiperplasias epiteliales, procesos inflamatorios crónicos, reflujo gastroesofágico, esófago de Barrett, entre otros ${ }^{9,10}$. El esófago de Barrett ocurre por el cambio del epitelio escamoso a tejido columnar metaplásico en el segmento distal del esófago, debido a un reflujo gastroesofágico severo. Existe un mayor riesgo de evolución a un adenocarcinoma de esófago ${ }^{11}$. El epitelio escamoso puede malignizarse por múltiples causas a un carcinoma de células escamosas o epidermoide. Representa cerca de un $90 \%$ de los casos de cáncer esofágico en Asia Oriental, pero en el Occidente y en especial en Chile, sólo representa el 50\%, por un importante aumento del adenocarcinoma esofágico ${ }^{12}, \mathrm{Su}$ incidencia en Chile es de 4 x 100.000 habitantes $^{13}$. Se postula una transformación neoplásica de las células epidermoides del esófago aperistáltico debido a la constante irritación de la mucosa por factores químicos y por mutaciones genéticas asociadas a la acalasia $^{14}$. Otra explicación radica en la fermentación de los alimentos retenidos en el esófago, lo cual produce un aumento de las poblaciones bacterianas intraesofágicas, con incremento de compuestos nitrogenados, por más de 10 años ${ }^{15}$, los cuales son altamente carcinogénicos ${ }^{16}$.

La relación objetiva entre acalasia y cáncer ha sido muy imprecisa debido a la variabilidad de los resultados de los estudios, el pequeño número de pacientes en el análisis estadístico, el poco seguimiento a través de los años y la presencia de factores de riesgo tales como tabaquismo y alcoholismo ${ }^{17}$. Este cáncer requiere alrededor de 10-20 años para su aparición desde los primeros síntomas de acalasia ${ }^{6,15-18}$. En la actualidad, ha habido varios trabajos que han intentado establecer el riesgo de padecer cáncer esofágico en pacientes con acalasia (Tabla 1$)^{15-22}$. Son 11 estudios, nueve de ellos con pacientes operados por vía laparotómica y los dos últimos ${ }^{23,24}$ por vía laparoscópica. Hay un número variable de pacientes incluidos, pero varios no reportan el porcentaje de seguimiento que tiene una trascendental importancia para los resultados. Se aprecia en la tabla que hay una clara correlación entre un mayor seguimiento y un mayor número de casos que desarrollan cáncer del esófago. El riesgo de aparición de esta neoplasia varía de 1/152 pacientes-año a 2.477 pacientes-año, lo que refleja una importante heterogeneidad de los reportes.

En el estudio realizado por Zendehdel ${ }^{15}$ se evidencia un alto riesgo que pacientes masculinos acalásicos padezcan carcinomas epidermoides $\mathrm{y}$, ocasionalmente, adenocarcinomas. Otro estudio realizado por Leewenburgh ${ }^{18}$ muestra que con el pasar de los años se acumula la incidencia del cáncer esofágico después del diagnóstico de acalasia, mostrando un incremento cercano al $10 \%$ tras 20 años de seguimiento. Respecto al abordaje laparoscópico, hay gran cantidad de publicaciones con seguimiento solo hasta los 5 años. Este hecho ha llevado a que no se han reportado casos con desarrollo de carcinoma esofágico, excepto en el estudio de Costantini ${ }^{23}$ en que señala la aparición en dos casos de 1.000 operados $(0,2 \%)$ con un seguimiento promedio de 5,5 años. Estos pacientes tuvieron el cáncer 8 y 14 años poscirugía. Nosotros hemos evaluado a 80 pacientes sometidos a cirugía laparoscópica con un seguimiento de 17 años, encontrando a 3 pacientes $(4,7 \%)$ con cáncer esofágico a los 9-12-15 años postoperados ${ }^{24}$.

\section{Conclusiones}

La acalasia esofágica es una enfermedad potencialmente preneoplásica, con una incidencia 
Tabla 1. Incidencia reportada de la acalasia y cáncer de esófago en los últimos estudios

\begin{tabular}{|c|c|c|c|c|c|c|}
\hline Estudio & $\begin{array}{c}\text { n de } \\
\text { pacientes } \\
\text { con } \\
\text { acalasia }\end{array}$ & $\begin{array}{l}\text { n de pacientes } \\
\text { que desarrollaron } \\
\text { cáncer esofágico } \\
\text { al seguimiento }\end{array}$ & $\begin{array}{l}\text { Tiempo del } \\
\text { seguimiento } \\
\text { (años) }\end{array}$ & $\begin{array}{l}\text { Ratio de incidencia } \\
\text { de cáncer esofágico } \\
\text { (1/pacientes - año) }\end{array}$ & $\begin{array}{c}\text { Aumento estimado } \\
\text { del riesgo de cáncer } \\
\text { esofágico }\end{array}$ & $\begin{array}{c}\text { Tiempo promedio } \\
\text { entre posoperatorio } \\
\text { y cáncer esofágico } \\
\text { (años) }\end{array}$ \\
\hline Peracchia, $1991^{20}$ & 244 & 1 & 3,7 & $1 / 1.200$ & 17 & 9 \\
\hline Aggestrup, $1992^{21}$ & 66 & 10 & 23,2 & $1 / 375$ & 27 & --- \\
\hline Brücher, $1998^{22}$ & 124 & 4 & 5,6 & $1 / 173$ & 140 & 32 \\
\hline Zendehdel, $2007^{15}$ & 2.896 & 22 & 9,9 & $1 / 1.170$ & 10 & --- \\
\hline Zaninotto, $2008^{16}$ & 228 & 4 & 18,3 & $1 / 705$ & 35 & --- \\
\hline Leeuwenburgh, $2010^{18}$ & 448 & 15 & 9,6 & $1 / 299$ & 28 & 24 \\
\hline
\end{tabular}

significativamente mayor de carcinoma esofágico, con un riesgo 7 a 140 veces mayor que la población general. Este aumento ocurre con 10 o más años después de la cirugía, por lo que es necesario realizar un seguimiento de estos pacientes con análisis subjetivo y objetivo (endoscopías seriadas) cada 5 años, para detectar lo más tempranamente posible las alteraciones de la mucosa esofágica que pueden derivar en un cáncer del esófago.

\section{Responsabilidades éticas}

Protección de personas y animales. Los autores declaran que para esta investigación no se han realizado experimentos en seres humanos ni en animales.

Confidencialidad de los datos. Los autores declaran que en este artículo no aparecen datos de pacientes.

Conflictos de interés: no hay.

\section{Bibliografía}

1. Richter JE. Oesophageal motility disorders. Lancet 2001;358:823-8. DOI: $10.1016 / \mathrm{S} 0140-6736(01) 05973-6$

2. Csendes A, Smok G, Braghetto I, González P, Henríquez A, Csendes P, et al. Histological studies of Auerbach's plexuses of the oesophagus, stomach, jejunum, and colon in patients with achalasia of the oesophagus: correlation with gastric acid secretion, presence of parietal cells and gastric emptying of solids. Gut 33;150-4. DOI: 10.1136/ gut.33.2.150.

3. Goyal RK, Chaudhury A. Physiology of normal esophageal motility. J Clin Gastroenterol. 2008;42:610-9.
DOI: 10.1097/MCG.0b013e31816b444d.

4. Fagge $\mathrm{CH}$. A case of simple stenosis of the esophagus followed by epithelioma. Guy's Hosp Rep. 1955; 17:413.

5. Csendes A, Braghetto I, Mascaró J, Henríquez A. Late subjective and objective evaluation of the results of esophagomyotomy in 100 patients with achalasia of the esophagus. Surgery 1988; 104:469-75. PMID: 3413676.

6. Csendes A, Braghetto I, Burdiles P, Korn $\mathrm{O}$, Csendes P, Henríquez A. Very late results of esophagomyotomy for patients with achalasia: clinical, endoscopic, histologic, manometric, and acid reflux studies in 67 patients for a mean follow-up of 190 months. Ann Surg. 2006;243:196-203. DOI: 10.1097/01. sla.0000197469.12632.e0

7. Heller E. Extramucose cardioplastik beim chrorischen cardiospasmus with dilatation der oesophagus. Mitteilunger ans den Grenzgelien Medizin und Chirurgie 1913;27:141-9.

8. Zaaijer JH. Cardiospasm in the aged. Ann Surg. 1923;77:615-7.

9. Agha FP, Keren DF. Barrett's esophagus complicating achalasia after esophagomyotomy. A clinical, radiologic, and pathologic study of 70 patients with achalasia and related motor disorders. $\mathrm{J}$ Clin Gastroenterol. 1987;9:232-7. PMID: 3571900.

10. Lehman MB, Clark SB, Ormsby AH, Rice TW, Richter JE, Goldblum JR. Squamous mucosal alterations in esophagectomy 
specimens from patients with endstage achalasia. Am J Surg Pathol. 2001;25:1413-8. DOI: 10.1097/00000478200111000-00009.

11. Schoofs N, Bisschops R, Prenen H. Progression of Barrett's esophagus toward esophageal adenocarcinoma: an overview, Ann Gastroenterol. 2017;30:1-6. DOI: 10.20524/aog.2016.0091.

12. Csendes A, Braghetto I, Cardenal G, Cortés S. Resultados de la esofagectomía en 53 pacientes con adenocarcinoma del esófago y Barrett extenso. Rev Chil Cir. 2013,65;64-74. https://dx.doi.org/10.4067/ S0718-40262013000200004.

13. Csendes A, Cuneo N, Figueroa M, Orellana O. Evolución de las operaciones por enfermedades del intestino proximal y glándulas anexas realizadas en Chile entre 2011 y 2016. Rev Cir.201;71:1117. http://dx.doi.org/10.4067/s245245492019000200111.

14. Manoel-Caetano Fda S, Borim AA, Caetano A. Cytogenetic alterations in chagasic achalasia compared to esophageal carcinoma. Cancer Genet Cytogenet. 2004;149:17-22. DOI: 10.1016/s01654608(03)00274-7.

15. Zendehdel K, Nyrén O, Edberg A, Ye W. Risk of esophageal adenocarcinoma in achalasia patients, a retrospective cohort study in Sweden. Am J Gastroenterol. 2007;106:57-61. DOI: 10.1038/ ajg.2010.449.

16. Zaninotto G, Rizzetto C, Zambon P, Guzzinati S, Finotti E, Costantini M. Long-term outcome and risk of oesophageal cancer after surgery for achalasia. Br J Surg. 2008;95:1488-94. DOI: 10.1002/bjs.6413

17. Meijssen MA, Tilanus HW, van Blankenstein M, Hop WC, Ong GL. Achalasia complicated by oesophageal squamous cell carcinoma: a prospective study in 195 patients. Gut 1992;33:155-8. DOI: 10.1136/gut.33.2.155.

18. Leeuwenburgh I, Scholten P, Alderliesten J, Tilanus HW, Looman CW, Steijerberg EW, et al. Long-term esophageal cancer risk in patients with primary achalasia: a prospective study. Am J Gastroenterol. 2010;105:2144-9. DOI: 10.1038/ ajg.2010.263.

19. Wychulis AR, Woolam GL, Andersen HA, Ellis FH Jr. Achalasia and carcinoma of the esophagus. JAMA 1971;215:1638-41. PMID: 5107684.

20. Peracchia A, Segalin A, Bardini R, Ruol A, Bonavina L, Baessato M. Esophageal carcinoma and achalasia: prevalence, incidence and results of treatment. Hepatogastroenterology 1991;38:514-6. PMID: 1778581.

21. Aggestrup S, Holm JC, Sørensen HR. Does achalasia predispose to cancer of the esophagus? Chest 1992;102:1013-6. DOI: 10.1378/chest.102.4.1013.

22. Brücher BL, Stein HJ, Bartels H, Feussner H, Siewert JR. Achalasia and esophageal cancer: incidence, prevalence, and prognosis. World J Surg. 2001;25:745-9. DOI: $10.1007 / \mathrm{s} 00268-001-0026-3$.

23. Costantini M, Salvador R, Capovilla G, Vallese L, Costantini A, Nicoletti L, et al. A Thousand and One Laparoscopic Heller Myotomies for Esophageal Achalasia: a 25-Year Experience at a Single Tertiary Center. J Gastrointest Surg. 2019;23:2335. doi: 10.1007/s11605-018-3956-x. Epub 2018 Sep 20.

24. Csendes A, Figueroa M, Orellana O, Lanzarini E, Panza B. Very long-term (17 years) subjective and objective evaluations of the durability of laparoscopic Heller myotomy in patients with Achalasia of the esophagus (90\% follow up); a real challenge to POEM. Surg Endosc. 2021 Jan 20. doi: 10.1007/ s00464-020-08273-1. Online ahead of print. 